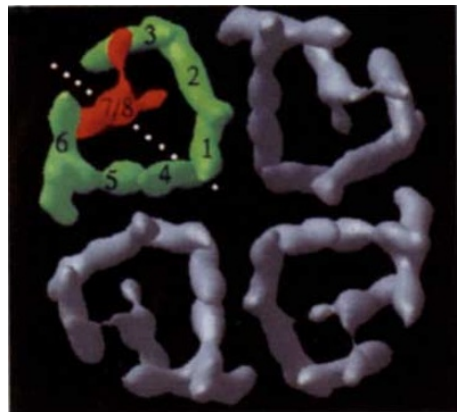

Fig. 1 Top view of the AQP1 tetramer'.

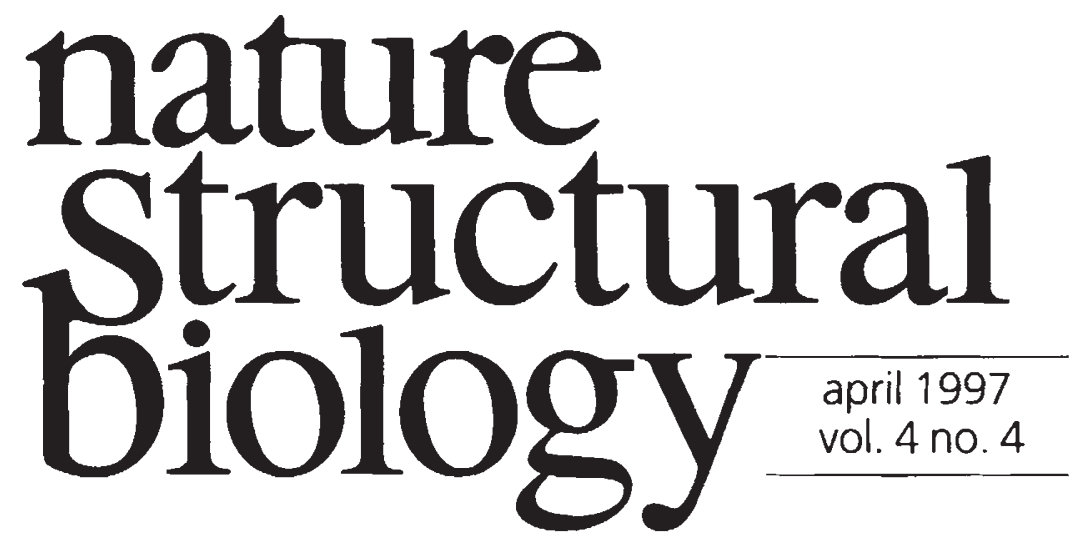

\section{A biological water filter}

Oil and water, as the saying goes, do not mix. So how do the many physiological processes that require the rapid, bulk movement of water-for example, the absorption of fluid in the guts of sap-sucking insects, the reabsorption of fluid in the kidney, the secretion of sweat, of tears, of bile and of amniotic fluid, transpiration in plants, and so on-cope the highly hydrophobic nature of cell membranes? Part of the answer (reported in this issue of Nature Structural Biology $y^{1}$ ) is provided by the structure of a member of a family of membrane channel proteins that is capable of transporting water-aquaporin 1 , determined at $\sim 6 \AA$ resolution using cryoelectron microscopy techniques.

\section{The water hole}

Movement of water across cell membranes occurs by two distinct pathways. The first pathway involves simple diffusion; the plasma membrane of all cells is moderately permeable to water (a biochemist might say that oil and water do not mix much), sufficiently so that the membrane can maintain osmotic equilibrium in static external environments. Some cells, however, have the ability to transport water across their cell membranes at greatly accelerated rates: for example, red blood cells, renal proximal tubules and the tonoplast of plant cell vacuoles. The high permeability of these tissues suggested the existence of a second water transport pathway, through a 'water channel'. This prediction was borne out with the discovery of CHIP28 (channel-forming integral membrane protein of $28 \mathrm{kDa}$ ) in the membranes of red blood cells and the water-permeable segments of the nephron.

CHIP28, now better know as aquaporin l (AQP1), was found to be a member of an ancient family of channel-forming proteins known as the MIP family, named after the first member to be isolated, the major intrinsic protein (MIP) of bovine lens fibres. Furthermore, AQP1 had all the characteristics of the predicted water channel in that it is a water selective, and osmotically driven, membrane pore. Many more aquaporins--a functionally defined subset of the MIP family that transport water--have since been isolated and characterized, including proteins found in bacteria, animals and plants ${ }^{2}$.

AQP1 is found in a variety of secretory and resorptive epithelia where it 




Fig. 2 The AQP1 monomer, seen from the side, reveals that the six outer helices appear to be arranged as two domains (helices 1-3 and 4-6) related by twofold symmetry, consistent with the observation of tandem repeats in the sequence of AQP1 (and the MIP family in general) ${ }^{1}$.

1. LI, H., Lee, S. \& Jap, B.K. Nature Struct. Biol. 4, 263-265 (1997)

2. Agre, P., Brown, D. \& Nielsen, S. Curr. Opin Cell. Biol. 7, 472-483 (1995)

3. Nielsen, S., Smith, B.L., Christensen, E.I. \& Agre, P. Proc. Natl. Acad. Sci. USA 90 7275-7279 (1993)

4. Preston, G.M., Smith, B.L., Zeidel, M.L. Moulds, J.J. \& Agre, P. Science 265, 1585-1587 (1994)

5. Deen, P.M.T. et al. Science 264, 92-95 (1994)

6. Jap, B.K. \& Li, H.L. J. Mol. Biol. 251, 413-420 (1995)

7. Mitra, A.K., van Hoek, A.N., Wiener, M.C. Verkman, A.S. \& Yeager, M. Nature Struct Biol. 2, 726-729 (1995)

8. Walz, T, Typke, D., Smith, B.L., Agre, P. \& Engel, A. Nature Struct. Biol. 2, 730-732 Engel,

9. Knepper, M.A. Proc. Natl. Acad. Sci. USA 91 6255-6258 (1994) has been suggested to confer high osmotic water permeability ${ }^{3}$. The channel is thought to be involved in a wide range of physiological processes, including: maintaining the desiccation and transparency of the lens and cornea in the anterior chamber of the eye; the secretion of the cerebrospinal fluid through the choriod epithelia; the formation and concentration of bile in the bile duct and gall bladder; the water permeability of the continuous endothelia of capillaries (indeed, its presence in the lung alveoli may explain the rapid water uptake and haemolysis of freshwater drownings). Surprisingly, despite these indications of involvement in water transport, mutations in the human aquaporin 1 gene, which knocks out the function of the protein, have no apparent phenotypic or clinical consequences, suggesting that its role is not as simple (or necessarily as central) as first thought ${ }^{4}$.

Whereas AQP1 seems to be constitutively present in many tissues, AQP2, which is essential for the regulation of body water balance, is expressed exclusively in renal collecting duct principal cells and is induced by the antidiruetic hormone vasopressin and, in the longer term, by water restriction $^{2}$. The presence of the hormone is thought to result in the targeted exocytosis of intracellular water channel-laden vesicles to the apical plasma membrane of the collecting duct cells. Mutations in aquaporin 2, unlike those in aquaporin 1, have dramatic consequences, resulting in a severe form of nephrogenic diabetes insipidus where the patient is unable to concentrate his/her urine, despite high circulating levels of vasopressin ${ }^{5}$.

\section{A cylindrical pore}

Although individual monomers of $\mathrm{AQP} 1$ are capable of transporting water, the protien is arranges as a tetramer in the membrane (Fig. 1). Sequence analysis predicted that AQP1, and other aquaporins as well as the members of the MIP family in general, would be $\alpha$-helical transmembrane proteins, with six membrane-spanning helices. Projection maps hinted that this was the case ${ }^{6-8}$ and the low resolution three-dimensional structure from $\mathrm{Li}$, Lee and Jap ${ }^{1}$ now provide a persuasive demonstration that this is indeed so. They see six long rod-like features, interpreted as helices, forming a cylindrical structure, partially enclosing a 'branched rod', which the authors cautiously suggest may represent a short $\alpha$-helix and/or a $\beta$-sheet (Fig. 2 ). The six 'helices' account for $\sim 50 \%$ of the total amino acid sequence consistent with spectroscopic analyses indicating that the channels consists of $\sim 40-50 \% \alpha$-helix and $\sim 15-40 \% \beta$-sheet.

While clearly a step on the pathway to an atomic resolution structure, many of the physical characteristics of the channel, inferred from the biophysical properties of the transport process ${ }^{9}$, are not revealed by the study. The low activation energy for transit through the channel suggests that water encounters polar groups similar to the polar environment of the bulk solvent. The pore's selectivity, and the exclusion of ions (but not protons) and small neutral molecules, such as urea, might be explained by a narrow pore radius, which would be smaller that the smallest impermeable solute (urea, for example, at $2 \AA$ ) but greater than that of water $(1.5 \AA$ ). But water is thought to pass through the channel as a continuous, unbroken single-file column of molecules (at a rate of 2-4 $\times 10^{9}$ molecules per second per channel): how then does the pore prevent protons jumping along the column of water, in the form of $\mathrm{H}_{3} \mathrm{O}^{+}$(a requirement if the kidney is to conserve water while excreting acid)? Higher resolution structures, that reveal the mode of action of the aquaporins, are eagerly awaited. 